\title{
BMJ Open Reduced prevalence of small-for- gestational-age and preterm birth for women of low socioeconomic position: a population-based cohort study comparing antenatal midwifery and physician models of care
}

Daphne N McRae, ${ }^{1}$ Patricia A Janssen, ${ }^{1}$ Saraswathi Vedam, ${ }^{2}$ Maureen Mayhew, ${ }^{1}$ Deborah Mpofu, ${ }^{3,4}$ Ulrich Teucher, ${ }^{5}$ Nazeem Muhajarine ${ }^{4}$

To cite: McRae DN, Janssen PA, Vedam S, et al. Reduced prevalence of small-forgestational-age and preterm birth for women of low socioeconomic position: a population-based cohort study comparing antenatal midwifery and physician models of care. BMJ Open 2018;8:e022220. doi:10.1136/ bmjopen-2018-022220

- Prepublication history and additional material for this paper are available online. To view these files, please visit the journal online (http://dx.doi. org/10.1136/bmjopen-2018022220).

Received 9 February 2018 Revised 5 June 2018 Accepted 29 August 2018

Check for updates

(C) Author(s) (or their employer(s)) 2018. Re-use permitted under CC BY-NC. No commercial re-use. See rights and permissions. Published by BMJ.

For numbered affiliations see end of article.

Correspondence to Dr Daphne N McRae; daphne.mcrae@ubc.ca

\section{ABSTRACT}

Objective Our aim was to investigate if antenatal midwifery care was associated with lower odds of smallfor-gestational-age (SGA) birth, preterm birth (PTB) or low birth weight (LBW) compared with general practitioner (GP) or obstetrician (OB) models of care for women of low socioeconomic position.

Setting This population-level, retrospective cohort study used province-wide maternity, medical billing and demographic data from British Columbia, Canada.

Participants Our study included 57872 pregnant women, with low socioeconomic position, who: were residents of British Columbia, Canada, carried a singleton fetus, had low to moderate medical/obstetric risk, delivered between 2005 and 2012 and received medical insurance premium assistance.

Primary and secondary outcome measures We report rates, adjusted $\mathrm{ORs}(\mathrm{aOR})$, and $95 \% \mathrm{Cls}$ for the primary outcome, SGA birth (<the 10th percentile), and secondary outcomes, PTB ( $<37$ weeks' completed gestation) and LBW $(<2500 \mathrm{~g})$.

Results Our sample included 4705 midwifery patients, 45114 GP patients and 8053 OB patients. Odds of SGA birth were reduced for patients receiving antenatal midwifery versus $\mathrm{GP}(\mathrm{aOR} 0.71,95 \% \mathrm{Cl} 0.62$ to 0.82$)$ or $\mathrm{OB}$ care (aOR $0.59,95 \% \mathrm{Cl} 0.50$ to 0.69 ). Odds of PTB were lower for antenatal midwifery versus $\mathrm{GP}$ (aOR $0.74,95 \% \mathrm{Cl}$ 0.63 to 0.86 ) or OB patients (aOR $0.53,95 \% \mathrm{Cl} 0.45$ to $0.62)$. Odds of LBW were reduced for midwifery versus GP (aOR $0.66,95 \% \mathrm{Cl} 0.53$ to 0.82 ) or OB patients (aOR 0.43 , $95 \% \mathrm{Cl} 0.34$ to 0.54 ).

Conclusion Antenatal midwifery care in British Columbia, Canada, was associated with lower odds of SGA birth, PTB and LBW, for women of low socioeconomic position, compared with physician models of care. Results support the development of policy to ensure antenatal midwifery care is available and accessible for women of low socioeconomic position. Future research is needed to determine the underlying mechanisms linking midwifery care to better birth outcomes for women of low socioeconomic position.

\section{Strengths and limitations of this study}

- This large, population-level cohort study $(n=57872)$ represented the majority of pregnant women with low socioeconomic position in British Columbia, Canada (2005-2012).

- The rigorous modelling approach controlled for correlation in outcomes at a family and community level.

- Findings are generalisable to other high-resource settings which offer similar, publicly funded midwifery services.

- The study was limited by self-selection of care provider which could have introduced differences between cohorts in social/health risks undocumented in the maternity record.

- Results included a post hoc analysis controlling for antepartum morbidity to assess the magnitude of self-selection bias.

\section{INTRODUCTION}

As established in the literature, women of low socioeconomic position (SEP) are more susceptible to poor infant birth outcomes compared with women of higher SEP. ${ }^{1}$ In response to this inequity, researchers have sought to determine if antenatal midwifery care could minimise the risk of adverse newborn outcomes for women of low SEP. In a 2016 scoping review of randomised trials and observational studies from high-resource countries (1990-2015), comparing antenatal midwifery versus physician-led care for women of low $\mathrm{SEP},{ }^{2}$ results indicated lower risk of preterm birth (PTB), ${ }^{3}$ low birth weight $(\mathrm{LBW})^{4}$ and/or very low birth weight $(\mathrm{VLBW})^{45}$ for midwives' patients in some studies (or subpopulations within studies), 
yet other studies indicated no significant difference in outcomes by provider type.$^{6-8}$ Almost all of these studies were limited by non-representative sampling, ${ }^{36} 7$ inadequate study power ${ }^{6-10}$ and/or failure to control for confounders. ${ }^{46}$ All but one study ${ }^{6}$ were conducted in the USA. Addressing these limitations, we conducted a large, population-level study among women of low SEP with low to moderate medical/obstetric risk to investigate if antenatal midwifery care was associated with lower odds of small-for-gestational-age (SGA) birth, PTB or LBW compared with general practitioner (GP) or obstetrician (OB) models of care.

\section{METHODS}

\section{Study design}

Using a retrospective cohort design we examined the association between antenatal models of care and odds of SGA birth, PTB or LBW among women of low SEP with low to moderate medical/obstetric risk. In British Columbia (BC), women with low to moderate perinatal risk are eligible for midwifery care. Model of care was ascertained using practitioners' antenatal service billing records. Women may have had an initial appointment with a GP if this was their preferred type of maternity provider, or because they were waitlisted for midwifery care, required an OB referral, or were unaware of the options for OB or midwifery care until the first prenatal appointment. Therefore, we did not classify patients' model of care by initial practitioner contact (intent to treat). Rather, patients were classified according to the type of practitioner providing all of their routine antenatal care, with allowance for one routine visit with another practitioner type. Aside from excluding all patients with high perinatal risk, patients with low to moderate perinatal risk and two or more practitioner types providing routine antenatal care were excluded from the study. None of the GP or midwifery patients included in the study had antenatal conditions recorded in the perinatal record requiring transfer to an $\mathrm{OB}$, nor did any $\mathrm{OB}$ patients have antenatal conditions recorded in the record rendering them ineligible for midwifery care.

In BC, GPs and OBs are compensated by the Ministry of Health for each antenatal visit whereas midwives are compensated according to partial or full trimester of care, regardless of the number of antenatal visits provided (see table 1). Antenatal care with a GP was defined as greater than or equal to three routine antenatal visits with a GP, and less than or equal to one routine antenatal visit with an $\mathrm{OB}$, or less than or equal to one partial trimester of midwifery care. Antenatal care with an OB was operationalised as greater than or equal to three routine antenatal visits with an $\mathrm{OB}$, and less than or equal to one routine antenatal visit with a GP, or less than or equal to one partial trimester of midwifery care. Antenatal midwifery care was operationalised as greater than or equal to two partial or full trimesters of midwifery care (equivalent to a minimum exposure of three routine antenatal physician visits), and less than or equal to one routine GP or OB antenatal visit. $\mathrm{OB}$ consultations were not included as routine antenatal visits.

\section{Setting}

In BC, women select their preferred type of maternity caregiver depending on practitioner availability and as appropriate to their need for specialist care. In rare instances women may have planned, shared care between a small pool of midwives and GPs. Midwifery care in the Canadian context is equivalent to case load midwifery care as it is practised in Australia, the UK and other European countries. Midwives provide holistic, continuity of care in which a midwife, or a small pool of midwives, known to a woman is/are available on call 24 hours a day. ${ }^{11}$ The midwifery model is relationship based with antenatal appointments lasting $30-60 \mathrm{~min}$ on average ${ }^{12}$ to facilitate counselling, education, emotional support and informed choice. ${ }^{11}$ When a midwifery patient has moderate perinatal risk, as outlined in the BC College of Midwives' guidelines ${ }^{13}$ midwives are required to consult with a physician (generally an OB) and if high-risk complications arise they will recommend a transfer to $\mathrm{OB}$ care.

While many GPs and some OBs function in a continuity of care, relationship-based model, the volume of need and fee-for-service funding model for physicians leads to shorter antenatal visits. Within the midwifery model, fees are all inclusive based on care and annual case loads are limited allowing for longer antenatal visits on average. ${ }^{11}$ All three types of providers follow the same schedule of antenatal visits.

\section{Outcomes}

Our outcome data for this study were obtained from the BC Perinatal Data Registry (PDR). ${ }^{14}$ Registry data were abstracted from hospital and home birth records. As well, International Statistical Classification of Diseases, Tenth Revision, Canada (ICD-10-CA) codes were imported to the PDR from the Canadian Institutes of Health Information Discharge Abstract Database. The PDR captures approximately $99 \%$ of all $\mathrm{BC}$ births with validation studies reporting a $97 \%$ accuracy rate over all data fields. ${ }^{15}$

The primary outcome variable was SGA birth $(<10$ th percentile) according to Kierans and colleagues' sex-specific birth weight charts. ${ }^{16}$ Secondary outcomes included PTB (<37 weeks' completed gestation) and LBW $(<2500 \mathrm{~g})$. LBW may be attributable to PTB, intrauterine growth restriction, or both and is reported here to facilitate comparison with other studies.

\section{Study sample}

Our study sample included women who: were residents of $\mathrm{BC}$, received antenatal midwifery, GP or OB care, carried a singleton fetus, had low to moderate medical/obstetric risk, delivered between 1 January 2005 and 31 December 2012, received medical insurance premium assistance and were not registered Status Indian. All women were classified as having low to moderate medical and obstetric 
Table 1 Characteristics of antenatal models of care in British Columbia

\begin{tabular}{|c|c|c|c|}
\hline & \multicolumn{3}{|l|}{ Antenatal care provider } \\
\hline & Midwife & General practitioner & Obstetrician \\
\hline & \multicolumn{3}{|l|}{ Total BC population } \\
\hline Delivery provider† & $14.0 \%$ & $32.5 \%$ & $51.2 \%$ \\
\hline Patient risk level & Low to moderate $\ddagger$ & Low to moderate & Low, moderate and high \\
\hline $\begin{array}{l}\text { Cost of services for BC } \\
\text { residents§ }\end{array}$ & $\begin{array}{l}100 \% \text { coverage by provincial } \\
\text { medical insurance }\end{array}$ & $\begin{array}{l}100 \% \text { coverage by provincial } \\
\text { medical insurance }\end{array}$ & $\begin{array}{l}100 \% \text { coverage by provincial } \\
\text { medical insurance }\end{array}$ \\
\hline \multirow[t]{2}{*}{ Practitioner's billing method } & $\begin{array}{l}\text { Per course of care, MWs } \\
\text { can bill for full care }(100 \%) \\
\text { or partial care ( } 40 \% \text { or } 60 \%) \\
\text { per trimester, depending on } \\
\text { patient transfer }\end{array}$ & Per ANC visit & Per ANC visit \\
\hline & \multicolumn{3}{|l|}{ Study populationๆ } \\
\hline $\begin{array}{l}\text { Average number of routine } \\
\text { ANC visits }\end{array}$ & 10.9 & 8.5 & 9.0 \\
\hline \multicolumn{4}{|l|}{ Delivery provider (\%) } \\
\hline MW & 77.6 & 0.5 & 0.2 \\
\hline GP & 2.5 & 68.3 & 3.1 \\
\hline OB & 18.2 & 26.1 & 93.9 \\
\hline Other & 1.7 & 5.0 & 2.8 \\
\hline
\end{tabular}

${ }^{*}$ Any involvement in ANC (2014/2015). ${ }^{36}$

†May differ from the ANC provider, preliminary data (2016/2017). ${ }^{37}$

$\ddagger$ Based on guidelines produced by the College of Midwives of BC. ${ }^{13}$

§Residents must be eligible for provincial medical insurance (ie, Canadian citizens or permanent residents).

IStudy population consisted of low-SEP women with low to moderate perinatal risk, 2005-2012, these data were unavailable for the total BC population.

ANC, antenatal care; BC, British Columbia; GP, general practitioner; MW, midwife; OB, obstetrician; SEP, socioeconomic position.

risk if they were eligible for midwifery care throughout the antenatal period according to guidelines produced by the College of Midwives of $\mathrm{BC}^{13}$ and expert advice from our clinical team members. Conditions rendering women ineligible for midwifery care included diseases of the blood, blood-forming organs or of the circulatory system, pre-existing hypertension or diabetes, liver disorders, tuberculosis, or malaria, as recorded in the maternity record, history of more than one PTB, more than two caesarean section deliveries, or more than two spontaneous abortions (prior to 20 weeks' completed gestation), or in the current pregnancy pre-eclampsia/eclampsia, placenta previa with haemorrhage, isoimmunisation, incompetent cervix, hyperemesis gravidarum with metabolic disturbance, or age less than 14 years. (See online supplementary appendix A for a complete description of inclusion/exclusion variables and ICD-10-CA codes.)

Because the key indicator used to assess low SEP, medical insurance premium assistance, was not available for Status women (they had their insurance premiums paid through Health Canada) they were excluded from the study. We operationalised low SEP as receipt of BC Medical Services Plan (MSP) regular premium subsidy assistance during the year of delivery. ${ }^{17}$ Eligibility for this assistance is based on family, net income ceiling exclusive of federal or provincial childcare or disability benefits. During the study period the ceiling ranged from \$24000-\$30000 for a family of three depending on the year of receipt. ${ }^{17}$ This is comparable to Statistics Canada's before-tax, low-income cut-off for a family of three (\$23 358-\$33933 as of 2008), which is a standard measure of poverty. ${ }^{18}$

\section{Sample size estimates}

During the study period women living in the poorest neighbourhood income quintiles in Canada experienced a $9.9 \%$ prevalence of SGA. ${ }^{19}$ To detect an absolute difference in prevalence of $3 \%$ (similar to estimates of prevalence in the general population) from a baseline of $9.9 \%$ we required a minimum sample of 1249 midwifery patients, 2497 OB patients and 4861 GP patients. Type I error was set at $\mathrm{p}=0.025$ two sided, and type II error set at 0.20 . 
We estimated $16.2 \%$ of the total $\mathrm{BC}$ population received MSP premium assistance, ${ }^{20}$ equivalent to 4154 midwifery patients and 36255 physician patients during the study period, excluding those who would not meet our criteria for low to moderate obstetrical risk. Sample size calculations were conducted using OpenEpi V.3.01.

\section{Statistical analyses}

To assess the association of model of care and SGA, PTB and LBW, we developed logistic regression models using a generalised estimating equation (GEE) approach. ${ }^{21}$ This method allowed for adjustment of variance estimates to accommodate potential correlation for women delivering multiple infants during the study period and for clustering of effects by community. ${ }^{21}$ Differing correlation structures were specified and compared using the Quasilikelihood under the Independence model Criteria (QIC) to determine the most appropriate correlation structure (the smaller the QIC the better the structure's fit). ${ }^{21}$ Binomial distributions were specified and models fitted with an exchangeable correlation structure (in which observations from the same cluster are assumed to be equally correlated) using logit link functions. ${ }^{21}$

We identified potential confounders, tested in our model, from the literature and based on our clinical experience. Variables analysed from the PDR included maternal age, parity, medical risk, prior obstetric risk, pre-pregnancy body mass index (BMI), infant sex, delivery year, smoking status, substance use, alcohol use, mental illness and northern residence. (See online supplementary appendix B for a complete list of covariate descriptions, data sources and ICD-10-CA codes.) From the Province of BC Statistics Division, we obtained socioeconomic rankings and income inequality rankings for each local health area (LHA) — 89 geographic and health administrative regions in $\mathrm{BC}$ that aggregate to larger Health Authorities. ${ }^{22}$ Income inequality rankings were based on the proportion of each LHA's total income from all households earning less than the median income compared with each LHA's total income from all households. In an entirely equitable LHA the poorest half of the households would garner $50 \%$ of the total income. ${ }^{22}$ We tested this variable as a potential confounder because it has been hypothesised that residence in an area with severe income inequality may increase the risk of poor self-concept potentially leading to lower commitment to pregnancy and unhealthy lifestyle choices. ${ }^{23}$ From the BC Ministry of Health we received data on women's neighbourhood income quintile, depending on residential postal code at delivery, ${ }^{24}$ and receipt of social assistance ${ }^{17}$ - public financial assistance granted to low-income individuals.

In logistic regression univariate analyses we identified variables that had Wald $\mathrm{X}^{2}$ values of $\mathrm{p}<0.25$ and retained these for our initial multivariable models. ${ }^{25}$ For the final variable selection we used a manual, backward elimination approach. Variables with a Wald $X^{2} p$ value $\geq 0.05$ were excluded from each multivariable model one at a time, beginning with the variable having the largest $\mathrm{p}$ value. ${ }^{25}$ After suspected confounders were removed from a model, coefficient estimates from models with and without the variable were examined to determine if the exclusion produced a greater than $20 \%$ change in any coefficient in the model. If this magnitude of change was detected, indicating a meaningful adjustment to (an) other variable(s), the eliminated variable was returned to the model (Hosmer, p92) ${ }^{25}$. This process was repeated until only variables meeting the criteria or those of clinical significance remained in the model. For births with no missing information we report unadjusted ORs and adjusted ORs (aORs) and 95\% CIs for SGA, PTB and LBW by model of care.

Lastly, we investigated residual confounding potentially arising from self-selection bias associated with pre-existing morbidity. If, for example, women chose OB care because of prior health conditions which were not documented in the PDR, then the OB cohort could have been comprised of systematically higher risk patients. To assess the potential effect of these conditions on our final models we conducted sensitivity analyses adjusting our final models for select antepartum morbidities (see definition in table 2). We also conducted sensitivity analyses excluding women with any known pre-existing conditions, to assess the impact of differing rates of moderate perinatal risk between cohorts on effect estimates. SAS Enterprise V.7.1 (SAS Institute) was used for data analysis.

\section{Patient involvement}

Patients were not involved in the development of the research question or study design. However, Canadian studies have shown that women of low SEP report more respectful care and greater autonomy in decision-making within the midwifery model compared with physician-led models of care ${ }^{12}{ }^{26}$ Results of this study may be of particular interest to women of low SEP who have a preference for midwifery care.

\section{RESULTS}

There were 4705 midwifery, $45114 \mathrm{GP}$ and 8053 OB pregnancies included in the study (figure 1). Both midwives and OBs' patients were, on average, older than GPs' patients, more likely to be multiparous, non-smokers and residing in urban areas (table 2). Although all women were of low income at a family level, a greater proportion of midwifery patients lived in wealthier towns/districts (LHAs) and neighbourhoods compared with GP or OB patients. This may be a reflection of health policy influencing the distribution of midwifery availability across the province. Midwifery care may be more available in desirable (ie, wealthier, southern, urban) areas as midwives are able to choose where they will open a practice and they are not eligible for the same financial incentives offered to rural and remote physicians. ${ }^{27}$

Midwifery and OB patients less frequently reported alcohol or substance use during pregnancy compared 
Table 2 Frequencies and proportions of maternal characteristics by antenatal model of care, British Columbia, 2005-2012 $(n=57872)$

\begin{tabular}{|c|c|c|c|}
\hline \multirow[b]{3}{*}{ Characteristics } & \multicolumn{3}{|c|}{ Antenatal model of care } \\
\hline & MW & GP & OB \\
\hline & $\mathrm{n}=4705(\%)$ & $n=45114(\%)$ & $\mathrm{n}=8053(\%)$ \\
\hline \multicolumn{4}{|l|}{ Age (years) } \\
\hline $14-19$ & $155(3.29)$ & $4697(10.41)$ & $338(4.20)$ \\
\hline $20-24$ & $893(18.98)$ & $14789(32.78)$ & $1447(17.97)$ \\
\hline $25-29$ & $1619(34.41)$ & $13161(29.17)$ & $2303(28.60)$ \\
\hline $30-34$ & $1362(28.95)$ & 7966 (17.66) & $2113(26.24)$ \\
\hline $35-39$ & $573(12.18)$ & $3730(8.27)$ & $1387(17.22)$ \\
\hline$\geq 40$ & $103(2.19)$ & $771(1.71)$ & $465(5.77)$ \\
\hline \multicolumn{4}{|l|}{ Parity* } \\
\hline Nullipara & $2177(46.27)$ & $23141(51.30)$ & 3617 (44.91) \\
\hline Multipara & $2528(53.73)$ & $21972(48.70)$ & $4435(55.07)$ \\
\hline Medical risk†‡ & $14(0.30)$ & $414(0.92)$ & $132(1.64)$ \\
\hline Prior obstetric risk†§ & $124(2.64)$ & $1669(3.70)$ & $478(5.94)$ \\
\hline Mental illness† & $1020(21.68)$ & $5146(11.41)$ & $610(7.57)$ \\
\hline Receiving social assistance $†$ & $310(6.59)$ & $5833(12.93)$ & $814(10.11)$ \\
\hline \multicolumn{4}{|c|}{ Pre-pregnancy body mass index (BMI) ${ }^{\star \star}$} \\
\hline Underweight & $229(4.87)$ & $2300(5.10)$ & $519(6.44)$ \\
\hline Normal & $2612(55.52)$ & $16777(37.19)$ & $2990(37.13)$ \\
\hline Overweight & $689(14.64)$ & $5829(12.92)$ & $877(10.89)$ \\
\hline Obese & $335(7.12)$ & $3792(8.41)$ & $479(5.95)$ \\
\hline Unknown & $840(17.85)$ & 16416 (36.39) & 3188 (39.59) \\
\hline \multicolumn{4}{|l|}{ Smoking status } \\
\hline Never & $992(21.08)$ & $6666(14.78)$ & $1868(23.20)$ \\
\hline Former & $690(14.67)$ & $5028(11.15)$ & $434(5.39)$ \\
\hline Current & $471(10.01)$ & $9910(21.97)$ & $800(9.93)$ \\
\hline Unknown & $2552(54.24)$ & $23510(52.11)$ & $4951(61.48)$ \\
\hline Substance use in pregnancy $\dagger, \dagger \dagger$ & $179(3.80)$ & $3273(7.25)$ & $302(3.75)$ \\
\hline Alcohol identified as a risk $\dagger$ & $57(1.21)$ & $1109(2.46)$ & $63(0.78)$ \\
\hline \multicolumn{4}{|l|}{ Utilisation of prenatal carełł } \\
\hline Intense & $98(2.08)$ & $304(0.67)$ & $60(0.75)$ \\
\hline Adequate & $1420(30.18)$ & $6851(15.19)$ & $902(11.20)$ \\
\hline Intermediate & $1927(40.96)$ & $19929(44.17)$ & 2601 (32.30) \\
\hline Inadequate & $273(5.80)$ & 6986 (15.49) & $980(12.17)$ \\
\hline Unknown & 987 (20.98) & $11044(24.48)$ & 3510 (43.59) \\
\hline Antepartum morbidity†§§ & 349 (7.42) & $6843(15.17)$ & 1955 (24.28) \\
\hline \multicolumn{4}{|l|}{ Delivery (year) } \\
\hline 2005 & 307 (6.52) & $5772(12.79)$ & 955 (11.86) \\
\hline 2006 & 437 (9.29) & $6028(13.36)$ & $1002(12.44)$ \\
\hline 2007 & $471(10.01)$ & $6133(13.59)$ & $1074(13.34)$ \\
\hline 2008 & $512(10.88)$ & $5892(13.06)$ & 977 (12.13) \\
\hline 2009 & $606(12.88)$ & $5640(12.50)$ & 910 (11.30) \\
\hline 2010 & $694(14.75)$ & $5371(11.91)$ & $1000(12.42)$ \\
\hline 2011 & 796 (16.92) & 5337 (11.83) & 1014 (12.59) \\
\hline
\end{tabular}


Table 2 Continued

\begin{tabular}{|c|c|c|c|}
\hline \multirow[b]{3}{*}{ Characteristics } & \multicolumn{3}{|c|}{ Antenatal model of care } \\
\hline & MW & GP & OB \\
\hline & $\mathrm{n}=4705(\%)$ & $\mathrm{n}=45114(\%)$ & $\mathrm{n}=8053(\%)$ \\
\hline 2012 & $882(18.75)$ & $4941(10.95)$ & $1121(13.92)$ \\
\hline \multicolumn{4}{|l|}{ Neighbourhood SEPףף } \\
\hline High & $624(13.26)$ & $4984(11.05)$ & $646(8.02)$ \\
\hline Low/medium & 4081 (86.74) & 40130 (88.95) & 7407 (91.98) \\
\hline \multicolumn{4}{|c|}{ Local health area (LHA) population demographic ${ }^{\star \star \star}$} \\
\hline Urban & $4548(96.66)$ & 42489 (94.18) & 7889 (97.96) \\
\hline Rural & $145(3.08)$ & $2576(5.71)$ & $145(1.80)$ \\
\hline Unknown & $12(0.26)$ & $49(0.11)$ & $19(0.24)$ \\
\hline \multicolumn{4}{|c|}{ LHA socioeconomic rank††† } \\
\hline High (best) & $2638(56.07)$ & $13287(29.45)$ & $4043(50.20)$ \\
\hline Medium & $1472(31.29)$ & 22011 (48.79) & $3197(39.70)$ \\
\hline Low & $582(12.37)$ & $9710(21.52)$ & $739(9.18)$ \\
\hline Unknown & $13(0.28)$ & $106(0.23)$ & $74(0.92)$ \\
\hline \multicolumn{4}{|c|}{ 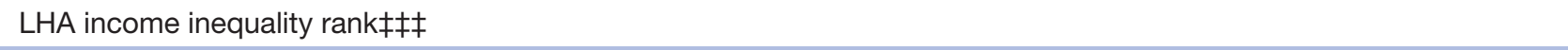 } \\
\hline High (worst) & $1667(35.43)$ & $10635(23.57)$ & $4177(51.87)$ \\
\hline Medium & $2326(49.44)$ & 25544 (56.62) & $3311(41.12)$ \\
\hline Low & $699(14.86)$ & $8841(19.60)$ & $530(6.58)$ \\
\hline Unknown & $13(0.28)$ & $94(0.21)$ & $35(0.43)$ \\
\hline Northern residence†£§§ & $136(2.89)$ & $6032(13.37)$ & $291(3.61)$ \\
\hline
\end{tabular}

All characteristics examined differed significantly by model of care $\left(X^{2} p<0.0001\right)$.

${ }^{*}$ Missing cases amount to five or less.

†Values represent cases classified as 'Yes', the remainder of the cases were classified as 'No', 'Unknown', or were undocumented.

fIncluded maternal disease of the respiratory or digestive system, and endocrine, nutritional or metabolic disease.

SIncluded women with at least one of the following conditions in past pregnancy: infant with major congenital anomaly, neonatal death, stillbirth or one preterm delivery.

IIncluded any of the following diagnoses prior to, or during the current pregnancy: anxiety disorder, depression, postpartum depression, bipolar disorder, other/unknown (including schizophrenic, mood and psychotic disorders).

${ }^{* *}$ Classified according to Health Canada's guidelines. ${ }^{38}$

††Heroin/opioids, cocaine, methadone, solvents, marijuana or other/unknown drugs used at any time during pregnancy, prescription or other drug use identified as a risk at any time during pregnancy.

$\ddagger \ddagger$ Classifications based on Kotelchuck Adequacy of Prenatal Care Utilization Index. ${ }^{39}$

$\S \S$ Included pregnancy-induced hypertension, gestational diabetes (whether or not insulin dependent), anaemia, intrauterine growth restriction, viral disease, infection and parasitic disease, placenta previa without haemorrhage, polyhydramnios or oligohydramnios, antepartum haemorrhage $\geq 20$ weeks, sexually transmitted infection or HIV, or premature separation of the placenta.

११Neighbourhood income quintiles were classified as low/medium (quintiles 1-4) versus high (quintile 5). ${ }^{24}$

${ }^{* * \star}$ Rural LHAs had a population $<10000$ people.

†††Calculated by the province of BC's Statistics Division (BC Stats), based on a range of social determinants of health-reflecting area-level economic and social processes, and policy decisions. ${ }^{22}$

$\ddagger \ddagger \ddagger$ Calculated by BC Stats. ${ }^{22}$

$\S \S \S A$ the time of delivery, normal residence in BC's Northern Health Authority.

GP, general practitioner; MW, midwife; OB, obstetrician; SEP, socioeconomic position.

with GP patients. A higher proportion of GP and OB patients had moderate medical risk and prior obstetric risk than midwifery patients, though midwifery patients had higher prevalence of reported mental illness during or prior to pregnancy (table 2). Midwife and GP patients had higher rates of overweight or obese BMI than OB patients. Midwives' patients also had higher prevalence of adequate attendance at prenatal care compared with physicians' patients.
Of all infants in our study, $7.09 \%$ were SGA, $6.50 \%$ were PTB and $3.32 \%$ were LBW (table 3). On average there was a significant reduction in unadjusted odds of SGA for midwifery versus GP patients (OR $0.67,95 \%$ CI 0.58 to 0.77 ) and midwifery versus $\mathrm{OB}$ patients (OR $0.55,95 \% \mathrm{CI}$ 0.47 to 0.64$)$. GP versus $\mathrm{OB}$ patients were also less likely to have an SGA infant (OR $0.81,95 \%$ CI 0.75 to 0.89 ). When controlling for maternal age, parity, pre-pregnancy BMI, infant sex, smoking status, substance use, mental 


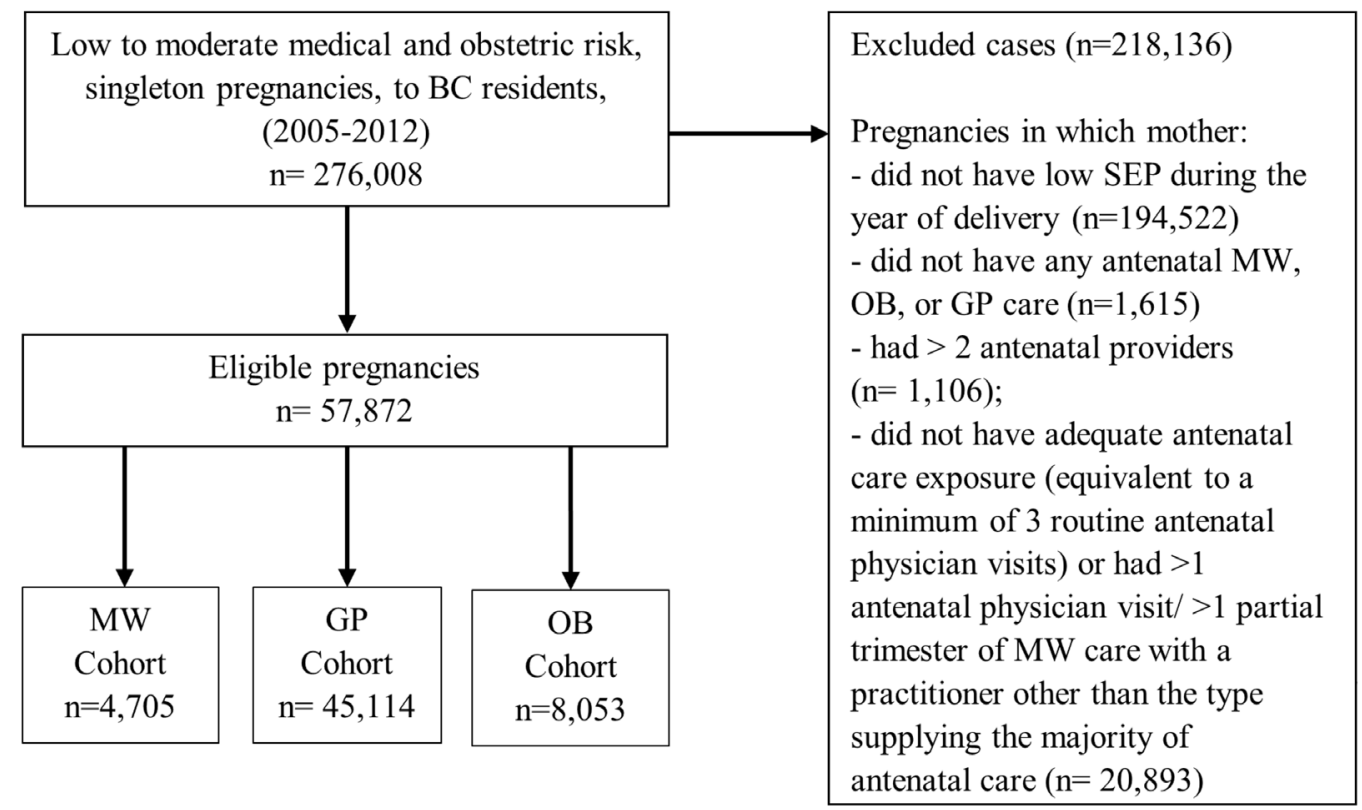

Figure 1 Eligibility flow chart. Total number of pregnancies meeting inclusion/exclusion criteria by cohort. BC, British Columbia; GP, general practitioner; MW, midwife; OB, obstetrician; SEP, socioeconomic position.

illness and LHA socioeconomic rank, women receiving antenatal care from midwives versus GPs had lower odds of having an SGA infant (aOR 0.71, 95\% CI 0.62 to 0.82 ) (table 3). Midwifery versus GP patients also had lower adjusted odds of SGA birth (aOR $0.59,95 \%$ CI 0.50 to 0.69). GP antenatal care was likewise associated with lower adjusted odds of SGA birth compared with OB care (aOR $0.83,95 \%$ CI 0.76 to 0.91 ).

The unadjusted odds of PTB were lower for woman receiving antenatal care from midwives versus GPs (OR $0.68,95 \%$ CI 0.59 to 0.79 ) and midwives versus OBs (OR $0.49,95 \% \mathrm{CI} 0.41$ to 0.57 ). GP versus $\mathrm{OB}$ patients also had lower unadjusted odds of PTB (OR $0.71,95 \%$ CI 0.65 to $0.78)$. When adjusting the PTB model for the same variables as the SGA model, as well as for medical risk, prior obstetric risk, delivery year, receipt of social assistance, alcohol use, neighbourhood SEP, LHA income inequality and northern residence, odds of PTB remained statistically significantly lower for midwifery versus GP care (aOR $0.74,95 \%$ CI 0.63 to 0.86 ) and midwifery versus $\mathrm{OB}$ care (aOR $0.53,95 \%$ CI 0.45 to 0.62 ). On average, GP patients also had lower adjusted odds of PTB compared with OB patients (aOR $0.72,95 \%$ CI 0.65 to 0.79 ).

Women receiving antenatal midwifery care had lower unadjusted odds of LBW compared with those in the care of GPs (OR $0.60,95 \%$ CI 0.49 to 0.74 ) or OBs (OR $0.39,95 \%$ CI 0.31 to 0.50$)$. GP versus $\mathrm{OB}$ patients also had lower unadjusted odds of LBW (OR $0.65,95 \%$ CI 0.58 to $0.73)$. After adjustment for maternal age, parity, prior obstetric risk, pre-pregnancy BMI, infant sex, smoking status and substance use, women in the care of midwives had lower odds of LBW compared with GP (aOR 0.66,

Table 3 Frequencies, proportions and adjusted ORs for small-for-gestational-age birth, preterm birth and low birth weight by antenatal model of care, British Columbia, 2005-2012

\begin{tabular}{|c|c|c|c|c|c|c|}
\hline & $\begin{array}{l}M W \\
n=4705\end{array}$ & $\begin{array}{l}\text { GP } \\
n=45114\end{array}$ & $\begin{array}{l}\text { OB } \\
n=8053\end{array}$ & MW versus GP & MW versus OB & GP versus OB \\
\hline & n (\%) & n (\%) & n (\%) & OR $(95 \% \mathrm{Cl})$ & OR $(95 \% \mathrm{Cl})$ & OR $(95 \% \mathrm{Cl})$ \\
\hline PTB† & $207 / 4702(4.40)$ & $2848 / 45028$ (6.32) & 698/8033 (8.69) & $0.74(0.63$ to 0.86$)$ & $0.53(0.45$ to 0.62$)$ & $0.72(0.65$ to 0.79$)$ \\
\hline LBW & $91 / 4704$ (1.93) & $1438 / 45091$ (3.19) & $393 / 8046(4.88)$ & $0.66(0.53$ to 0.82$)$ & $0.43(0.34$ to 0.54$)$ & 0.65 (0.58 to 0.74$)$ \\
\hline
\end{tabular}

All models adjusted for maternal age, parity, pre-pregnancy body mass index (BMI), infant sex, smoking status and substance use. *Model also adjusted for mental illness and local health area (LHA) socioeconomic rank. ORs based on 4095 births with SGA and 57722 total births with no missing information for this analysis.

†Model also adjusted for medical risk, prior obstetric risk, delivery year, receipt of social assistance, alcohol use, mental illness, neighbourhood socioeconomic position (SEP), LHA socioeconomic rank, LHA income inequality and northern residence. ORs based on 3753 PTB births and 57763 total births with no missing information for this analysis.

¥Model also adjusted for prior obstetric risk. ORs based on 1922 births with LBW and 57841 total births with no missing information for this analysis.

GP, general practitioner; LBW, low birth weight; MW, midwife; OB, obstetrician; PTB, preterm birth; SGA, small-for-gestational-age. 
$95 \%$ CI 0.53 to 0.82 ) or OB patients (aOR 0.43 , 95\% CI 0.34 to 0.54 ). GP patients also had lower adjusted odds of LBW compared with OB patients (aOR $0.65,95 \%$ CI 0.58 to 0.74$)$.

When testing for residual confounding by controlling for select antepartum morbidities the associations between model of care and SGA, PTB and LBW were attenuated but remained statistically significant (see online supplementary appendix C: table 1 ). Sensitivity analyses excluding women with prior medical risk or a history of obstetric risk (see table 2 for definitions) produced results nearly identical to our final models (see online supplementary appendix C: table 2).

\section{DISCUSSION}

\section{Strengths and weaknesses}

Our study demonstrated a statistically significant reduction in odds of SGA, PTB and LBW for infants born to women of low SEP receiving antenatal midwifery versus physician-led care in BC, Canada. This study represented the majority of pregnant, low-SEP women in BC during the study period, had adequate study power and tested a wide range of individual and area-level potential confounders. In addition, GEE logistic regression modelling allowed us to account for correlation in outcomes at a family and community level, a more rigours modelling approach than the methods used in previous studies. As this was a large, population-based study, findings are generalisable for other high-resource countries which offer similar, publicly funded midwifery services.

Our study was limited by its observational design. As women have been shown to refuse randomisation to retain choice in maternity care provision, ${ }^{28}$ and because midwifery care is a newer, government-funded maternity care option in BC (since 1998) in growing demand, evidence for causality will need to be established by repeated observational studies with representative samples over time. This study was also limited by a lack of data on the use of universal, objective screening tools for alcohol/substance use and mental health conditions, and it did not include measures of severity. In addition, there were no data available on race/ethnicity, language or culture, and we were not able to assess outcomes among women who were Status Indians. It should also be noted that in some cases antenatal midwifery and GP care included discussion or consultation with OBs for complex cases, and included transfer of care to OBs during labour and delivery when indicated. Though unmeasured, the quality of collaboration between practitioners and the use of obstetric referral will have had an influence on the results.

Women in the study self-selected their care provider, therefore it is possible that those with higher perinatal risk (on the low to moderate risk spectrum) chose OB care, creating a higher risk OB cohort. However, we did control for a wide range of known medical and obstetric risk factors when indicated, and overall the population had very low prevalence of known pre-existing risk (medical risk $0.97 \%$, prior obstetric risk $3.92 \%$ ). In addition, when we conducted two sensitivity analyses, controlling for antepartum morbidity (online supplementary appendix C: table 1), and second excluding patients with prior medical or obstetric risk (online supplementary appendix C: table 2), the main associations remained significant. Lastly, because women utilising midwifery care in BC may need to be proactive in ascertaining services early in pregnancy due to high demand, it is plausible that women who secured midwifery care were more knowledgeable about the healthcare system, more invested in their health or had greater ability to pursue preferred healthcare services. These skills, attitudes and values could have systematically differed between cohorts. Nonetheless, we did control for smoking, alcohol and pre-pregnancy BMI, which may reflect women's attitudes, beliefs and values during pregnancy, and this may have minimised self-selection bias.

\section{Results in comparison with other studies}

Our results for PTB coincide with a 2016 Cochrane review synthesising the findings of eight randomised controlled trials (RCTs) testing midwifery-led continuity models of care versus other models, including midwifery-physician models and medical-led care. In this review, the authors found a $24 \%$ reduction in risk of PTB, less than 37 weeks' gestation, for midwifery patients (average risk ratio 0.76 , $95 \%$ CI 0.64 to $0.91, \mathrm{n}=13238) .{ }^{29}$ This is comparable to our $26 \%$ reduction in odds of PTB, less than 37 weeks' gestation, for midwifery versus GP patients (aOR 0.74, $95 \%$ CI 0.63 to $0.86, \mathrm{n}=49819)$. As recommended in the Cochrane review, our study specifically focused on vulnerable women. Observational studies with non-representative samples (a free-standing birth centre serving primarily low-income African-American women, ${ }^{3}$ and an Australian, hospital-based cohort study restricted to women $<21$ years of age ${ }^{30}$ ) have also reported findings similar to ours. In an RCT for low-SEP women who had high risk of delivering LBW infants, odds of VLBW was significantly lower among a subgroup of African-American nurse-midwifery patients versus $\mathrm{OB}$ patients (OR $0.35,95 \%$ CI 0.1 to 0.9$).{ }^{5}$ However, there was no difference in odds of LBW or VLBW by practitioner type in the overall sample. Additionally, in a retrospective cohort study ${ }^{4}$ comparing outcomes of nurse-midwifery care to usual care for Medicaid recipients or uninsured patients residing in Westchester County, New York, nurse-midwifery patients had significantly lower risk of LBW and VLBW. Yet, in this study there was no adjustment for pre-existing health complications or perinatal risk which may have introduced bias.

Five other midwifery/physician studies involving women of low SEP have reported no significant differences in SGA or PTB by provider type. ${ }^{6-10}$ Almost all studies were limited by failure to control for pre-existing medical/obstetric risk ${ }^{6}$ or inadequate power to detect clinically important differences between cohorts. ${ }^{6-10}$ In one 
adequately powered, prospective cohort study $(\mathrm{n}=2957)^{7}$ comparing collaborative birth centre care provided by midwives (with $\mathrm{OB}$ referral for complications) versus $\mathrm{OB}$ or $\mathrm{OB}$ resident care, no statistically significant differences were reported. This study, however, was conducted in the USA and comprised $77 \%$ Hispanic women.

\section{Experience of antenatal care across models}

In our study, adequate antenatal care utilisation may have been a mechanism linking midwifery care to reduced odds of SGA, PTB and LBW. Midwives' patients had 2.3 times greater odds of adequately utilising antenatal care compared with GPs' patients and 2.5 times greater odds compared with OBs' patients. As revealed in a 2009 qualitative metasynthesis, antenatal care use by marginalised women is associated with their perception of their clinician's trustworthiness, cultural sensitivity and respect for life experience. ${ }^{31}$ Adequate use of antenatal care has been shown to protect against PTB, stillbirth and neonatal and infant death. ${ }^{32}$ If midwifery's relationship-based model of care encouraged antenatal care uptake, it may have indirectly affected prevalence of infant morbidity for women of low SEP.

Lack of patient trust may also have inhibited patient disclosure of compromising health conditions. Midwifery patients had higher prevalence of mental illness overall and for each category (ie, depression, anxiety, bipolar disorder) compared with GP or OB patients. Midwives' patients had a 2.2-fold increase in odds of documented mental illness, compared with GPs' patients and a 3.4-fold increase compared with OBs' patients. In our study, prevalence of depression for midwifery patients approximated that reported in the literature. In a review of 16 antenatal and postnatal depression studies $(n=35419)$ which were published between 2000 and 2016, and mainly conducted in western Europe, researchers reported a mean antenatal depression prevalence of $17.2 \% .{ }^{33}$ In our study, data on depression were collected between 2008 and 2012. The proportion of midwifery patients with depression prior to or during pregnancy was $18.8 \%$ in contrast to $12.8 \%$ for GP patients and $7.4 \%$ for $\mathrm{OB}$ patients.

Greater disclosure of sensitive information to midwives providing case load midwifery care has been noted in other studies. In the Australian midwifery cohort study previously cited, young women receiving case load midwifery care were significantly $(\mathrm{p}<0.01)$ more likely to report a history of mental illness, illicit drug use and involvement with the Department of Child Safety than those receiving standard maternity care. ${ }^{30}$ Likewise, in a small retrospective cohort study $(\mathrm{n}=194)$ conducted in the UK researchers examined birth outcomes by case load midwifery care to standard maternity care for women with vulnerabilities (ie, experiencing 'domestic violence, homelessness, mental health issues, substance and/or alcohol abuse' (Rayment-Jones, p411)) ${ }^{34}$. Women in the case load midwifery cohort were statistically significantly more likely to receive a referral to psychiatric care and/or domestic violence or other support services which may be indicative of higher rates of disclosure among midwifery patients. Of note, in both of these studies patients in the case load midwifery cohorts had either a higher mean number of antenatal appointments ${ }^{34}$ or a lower percentage of inadequate prenatal utilisation of care $(<5$ visits $) .{ }^{30}$ This likely increased clinician-patient familiarity which is a component of trust shown to influence domestic abuse disclosure. ${ }^{35}$

In our study, odds of antepartum morbidity were lower for midwives versus physicians' patients providing another clue as to the mechanisms linking midwifery care to a reduction in prevalence of SGA, PTB and LBW. Midwifery versus GP patients had 59\% lower odds of antepartum morbidity (see definition in table 2), and midwifery versus OB patients had $74 \%$ lower odds. When controlling for antepartum morbidity odds of SGA, PTB and LBW by model of care were attenuated but remained statistically significant (online supplementary appendix C: table 1 ). This suggests that even if antepartum morbidity were related to baseline differences in health status (selection bias), this could only partially explain the lower odds of adverse infant birth outcomes for women in the care of midwives versus physicians. It is plausible longer appointment times and a holistic approach to care may have made it possible for midwives to identify premorbid conditions (ie, borderline hypertension or anaemia) earlier in pregnancy and implement preventive measures before conditions progressed to antepartum morbidity.

\section{Implications}

Study findings indicate a need for policy which supports midwifery availability and accessibility for women of low SEP. This could include incentivising midwifery outreach to vulnerable populations by compensating midwives for the extra time involved in caring for women with higher socioeconomic risk. It could also mean increasing the volume of midwives practising in the province to meet current demand, and conducting targeted public awareness campaigns to educate low-SEP women about the government-funded options available in maternity care. Future studies are needed to identify which attributes of midwifery care influence infant birth outcomes for women of low SEP and the mechanisms (ie, physiological, psychological and/or behavioural) underlying this association. In our study midwifery care was associated with the lowest odds of adverse birth outcomes followed by GP, then OB care. Antenatal midwifery and GP practice may have greater similarity (with respect to continuity in care, provision of emotional support and volume of medical intervention) than midwifery to $\mathrm{OB}$ care. Therefore, it could be useful to analyse the characteristics of practice common to midwifery and GP care but which differ from OB practice.

\section{CONCLUSION}

Our study demonstrated lower odds of SGA birth, PTB and LBW for women of low SEP in BC who received antenatal midwifery versus physician-led care. As this was a 
large, population-based study with adequate study power and control for confounders, our results are generalisable to other high-resource countries offering similar midwifery services. Results of this study support the development of policy to ensure antenatal midwifery care is available and accessible for women of low SEP. Further research is needed to determine the mechanisms linking antenatal midwifery care to better birth outcomes among women of low SEP.

\section{Author affiliations}

${ }^{1}$ School of Population and Public Health, University of British Columbia, Vancouver, British Columbia, Canada

${ }^{2}$ Birth Place Lab, Department of Family Practice, University of British Columbia, Vancouver, British Columbia, Canada

${ }^{3}$ Saskatoon City Hospital, Saskatoon Health Region, Saskatoon, Saskatchewan, Canada

${ }^{4}$ Department of Community Health and Epidemiology, University of Saskatchewan, Saskatoon, Saskatchewan, Canada

${ }^{5}$ Department of Psychology, University of Saskatchewan, Saskatoon, Saskatchewan, Canada

Acknowledgements We thank Celeste Bickford for assistance in linking the Research Extracts and data preparation.

Contributors DNM designed the study, conducted the statistical analyses, interpreted the results, drafted the initial manuscript and revised subsequent drafts. NM and PAJ designed the study, reviewed the statistical analyses and interpreted the results. SV, MM and DM contributed to study design and clinical interpretation. UT contributed to interpretation. NM, PAJ, SV, MM, DM and UT reviewed and revised the manuscript. All authors approved the final manuscript.

Funding DNM was supported by an Arthur Smyth Scholarship from the College of Medicine, and a PhD Scholarship from the Department of Community Health and Epidemiology, University of Saskatchewan, Saskatoon, SK, Canada. PAJ is supported by a Senior Scholar salary award from the BC Children's Hospital Research Institute, Vancouver, BC, Canada.

Disclaimer All inferences, opinions and conclusions drawn in this study are those of the authors, and do not reflect the opinions or policies of the Data Stewards. Funding sources had no involvement in the study; the authors are independent of all funders.

Competing interests As of May 2018, DNM has been providing consulting services to the Midwives Association of BC. No other authors have competing interests to declare.

Patient consent Not required.

Ethics approval University of Saskatchewan, Biomedical Research Ethics Board (registration numbers 00001471 and 00008358) and the University of British Columbia, Children's and Women's Health Center of BC Research Ethics Board (registration number H14-01629).

Provenance and peer review Not commissioned; externally peer reviewed.

Data sharing statement № additional data available.

Open access This is an open access article distributed in accordance with the Creative Commons Attribution Non Commercial (CC BY-NC 4.0) license, which permits others to distribute, remix, adapt, build upon this work non-commercially, and license their derivative works on different terms, provided the original work is properly cited, appropriate credit is given, any changes made indicated, and the use is non-commercial. See: http://creativecommons.org/licenses/by-nc/4.0/.

\section{REFERENCES}

1. Blumenshine P, Egerter S, Barclay CJ, et al. Socioeconomic disparities in adverse birth outcomes: a systematic review. Am J Prev Med 2010;39:263-72.

2. McRae DN, Muhajarine N, Stoll K, et al. Is model of care associated with infant birth outcomes among vulnerable women? A scoping review of midwifery-led versus physician-led care. SSM Popul Health 2016;2:182-93.
3. Benatar S, Garrett AB, Howell E, et al. Midwifery care at a freestanding birth center: a safe and effective alternative to conventional maternity care. Health Serv Res 2013;48:1750-68.

4. Visintainer PF, Uman J, Horgan K, et al. Reduced risk of low weight births among indigent women receiving care from nurse-midwives. $J$ Epidemiol Community Health 2000;54:233-8.

5. Heins $\mathrm{HC}$, Nance NW, McCarthy BJ, et al. A randomized trial of nurse-midwifery prenatal care to reduce low birth weight. Obstet Gynecol 1990;75(3 Pt 1):341-5.

6. Simonet F, Wilkins R, Labranche E, et al. Primary birthing attendants and birth outcomes in remote Inuit communities--a natural "experiment" in Nunavik, Canada. J Epidemiol Community Health 2009;63:546-51.

7. Jackson DJ, Lang JM, Swartz WH, et al. Outcomes, safety, and resource utilization in a collaborative care birth center program compared with traditional physician-based perinatal care. Am J Public Health 2003;93:999-1006.

8. Cragin LE. Comparisons of care by nurse-midwives and obstetricians: birth outcomes for moderate risk women [Ph.D. thesis] Ann Arbor: University of California, San Francisco, 2002.

9. Fischler NR, Harvey SM. Setting and provider of prenatal care: association with pregnancy outcomes among low-income women. Health Care Women Int 1995;16:309-21.

10. McLaughlin FJ, Altemeier WA, Christensen MJ, et al. Randomized trial of comprehensive prenatal care for low-income women: effect on infant birth weight. Pediatrics 1992;89:128-32.

11. College of Midwives of British Columbia (CMBC), 2013. Midwifery model of practice: CMBC http://www.cmbc.bc.ca/pdf.shtml? Registrants-Handbook-11-05-Midwifery-Model-of-Practice (cited 22 Apr 2017).

12. Vedam S, Stoll K, Martin K, et al. Changing Childbirth in BC Steering Council. The Mother's Autonomy in Decision Making (MADM) scale: patient-led development and psychometric testing of a new instrument to evaluate experience of maternity care. PLoS One 2017;12:e0171804.

13. College of Midwives of British Columbia (CMBC), 2014. Indications for discussion, consultation and transfer of care: CMBC http:// www.cmbc.bc.ca/Registrants-Handbook-12-01-Indications-forDiscussion-Consultation-and-Transfer-of-Care.pdf (cited 2 May 2017).

14. Perinatal Services BC [creator] (2016), 2014. British Columbia perinatal data registry. Population Data BC [publisher]. Data Extract. PSBC http://www.perinatalservicesbc.ca/healthprofessionals/data-surveillance/perinatal-data-registry. (cited $6 \mathrm{Apr}$ 2017).

15. British Columbia Perinatal Health Program. British Columbia perinatal database registry annual report. Vancouver, BC: Perinatal Services $\mathrm{BC}, 2007$.

16. Kierans W, Kramer M, Wilkins R, et al. Charting birth outcome in British Columbia: determinants of optimal health and ultimate riskan expansion and update. Vancouver, BC: British Columbia Vital Statistics Agency, 2008.

17. British Columbia Ministry of Health [creator] (2016), 2014. Medical Services Plan (MSP) Payment Information File. V2. Population Data BC [publisher]. Data Extract. MOH. http://www.popdata.bc.ca/data (cited 6 Apr 2017).

18. Statistics Canada. Low income cut-offs for 2008 and low income measures for 2007. Ottawa, ON: Statistics Canada, 2009.

19. Canadian Institute of Health Information (CIHI). Too early, too small: a profile of small babies across Canada. Ottawa, ON: ClHI, 2009.

20. Government of British Columbia, 2015. Information release: freedom of information request - HTH-2015-50271 http://www.openinfo.gov. bc.ca/ibc/search/detail.page?config=ibc\&P110=recorduid:HTH2015-50271\&title=FOI\%20Request\%20-\%20HTH-2015-50271 (cited 12 Dec 2015).

21. SAS Institute Inc. SAS/STAT® 14.3 User's Guide: The GEE procedure. Cary, NC: SAS Institute Inc, 2017.

22. BC Stats. Socio-economic indices: local health area indices: Government of BC. 2013 https://www2.gov.bc.ca/gov/content/data/ statistics/people-population-community/socio-economic-profilesindices/socio-economic-indices (cited 2017 Dec 12).

23. Meng G, Thompson ME, Hall GB. Pathways of neighbourhood-level socio-economic determinants of adverse birth outcomes. Int $J$ Health Geogr 2013;12:32.

24. British Columbia Ministry of Health [creator] (2015), 2014. Consolidation File (MSP Registration \& Premium Billing). Population Data BC [publisher]. Data Extract. MOH http://www.popdata.bc.ca/ data (cited 6 April 2017).

25. Hosmer DW, Lemeshow S, Sturdivant RX. Applied logistic regression. Hoboken, N.J: Wiley, 2013. 
26. Vedam S, Stoll K, Rubashkin N, et al. CCinBC Steering Council. The Mothers on Respect (MOR) index: measuring quality, safety, and human rights in childbirth. SSM Popul Health 2017;3:201-10.

27. Government of B.C. Rural practice programs: Government of B.C.; 2018 https://www2.gov.bc.ca/gov/content/health/practitionerprofessional-resources/physician-compensation/rural-practiceprograms. (cited 2018 May 24).

28. Allen J, Stapleton H, Tracy S, et al. Is a randomised controlled trial of a maternity care intervention for pregnant adolescents possible? An Australian feasibility study. BMC Med Res Methodol 2013;13:138

29. Sandall J, Soltani H, Gates S, et al. Midwife-led continuity models versus other models of care for childbearing women. Cochrane Database Syst Rev 2016;4:CD004667.

30. Allen J, Gibbons K, Beckmann M, et al. Does model of maternity care make a difference to birth outcomes for young women? A retrospective cohort study. Int J Nurs Stud 2015;52:1332-42.

31. Downe S, Finlayson K, Walsh D, et al. 'Weighing up and balancing out': a meta-synthesis of barriers to antenatal care for marginalised women in high-income countries. BJOG 2009;116:518-29.

32. Partridge S, Balayla J, Holcroft CA, et al. Inadequate prenatal care utilization and risks of infant mortality and poor birth outcome: a retrospective analysis of $28,729,765$ U.S. deliveries over 8 years. $A m$ $J$ Perinatol 2012;29:787-93.

33. Underwood L, Waldie K, D'Souza S, et al. A review of longitudinal studies on antenatal and postnatal depression. Arch Womens Ment Health 2016;19:711-20.

34. Rayment-Jones H, Murrells T, Sandall J. An investigation of the relationship between the caseload model of midwifery for socially disadvantaged women and childbirth outcomes using routine data--a retrospective, observational study. Midwifery 2015;31:409-17.

35. Liebschutz J, Battaglia T, Finley E, et al. Disclosing intimate partner violence to health care clinicians - what a difference the setting makes: a qualitative study. BMC Public Health 2008;8:229.

36. Perinatal Services BC (PSBC). Midwifery report. Deliveries in BC. Vancouver, BC: PSBC, 2016.

37. Perinatal Services BC (PSBC). Annual Report 2016/17. Vancouver, BC: PSBC, 2017

38. Health Canada. Prenatal nutrition guidelines for health professionals: gestational weight gain. Ottawa, ON: Health Canada, 2014.

39. Kotelchuck M. An evaluation of the Kessner Adequacy of Prenatal Care Index and a proposed Adequacy of Prenatal Care Utilization Index. Am J Public Health 1994:84:1414-20. 